\title{
Vitamin D and diabetic foot ulcer: a systematic review and meta-analysis
}

\author{
Jiezhi Dai ${ }^{1}$, Chaoyin Jiang ${ }^{1}$, Hua Chen ${ }^{1}$ and Yimin Chai ${ }^{1}$
}

\begin{abstract}
We aimed to evaluate the association between vitamin D deficiency and diabetic foot ulcer (DFU) in patients with diabetes. Pubmed, EMBASE, BIOSIS, the Cochrane Library, and Web of Knowledge, last updated in July 2018, were searched. We assessed eligible studies for the association between vitamin D deficiency and DFU in diabetic patients. The mean difference (MD) or the odds ratio (OR) was calculated for continuous or dichotomous data respectively. Data were analyzed by using the Cochrane Collaboration's RevMan 5.0 software. Seven studies that involved 1115 patients were included in this study. There were significantly reduced vitamin D levels in DFU (MD $-13.47 \mathrm{nmol} / \mathrm{L}, 95 \% \mathrm{Cl}$ -16.84 to $\left.-10.10 ; P=0.34, P^{2}=12 \%\right)$. Severe vitamin $D$ deficiency was significantly associated with an increased risk of DFU (OR 3.22, 95\% Cl 2.42-4.28; $P=0.64, P^{2}=0 \%$ ). This is the first meta-analysis demonstrating the association between serum vitamin D levels and DFU. Severe vitamin D deficiency is significantly associated with an increased risk of DFU.
\end{abstract}

\section{Introduction}

The diabetic foot ulcer (DFU) is a severe complication in patients with diabetes mellitus $(\mathrm{DM})^{1}$. Patients with DFU have a higher mortality compared with diabetic patients without foot ulcer. Daousi et al. ${ }^{2}$ reported the mortality rate of DFU is about twice that of nonulcerated diabetic patients. However, there is a paucity of information that explores possible contributory factors on DFU.

In recent years, there is an increasing interest in the beneficial role of vitamin D in DM. Some researches have reported its effect on T-cell-mediated immunity, pancreatic insulin secretion and action as well as cell growth and healing ${ }^{3}$. Yakob et al. ${ }^{4}$ found the low levels of vitamin D may be related to the development of diabetic foot infections. Findings from in vitro studies reported that vitamin D could restore the production of antimicrobial peptides in primary cell from DFU and

Correspondence: Hua Chen (chuadr@aliyun.com) or Yimin Chai (ymchai@sjtu. edu.cn)

'Department of Orthopedic Surgery, Shanghai Jiao Tong University Affiliated Sixth People's Hospital, Shanghai, China

These authors contributed equally: Jiezhi Dai, Chaoyin Jiang. improve the in vitro wound-healing assays ${ }^{5}$. In rats, the topical application of vitamin D accelerated wound healing in a dose-dependent manner ${ }^{6}$. Another study found that calcitriol could promote endothelial and keratinocyte cell migration in a DFU model ${ }^{7}$. Thus, findings from previous studies showed the possible beneficial effects of vitamin D on wound healing in DFU. However, there were no conclusive results available. In this study, we conducted a review and meta-analysis of cohort studies to assess the relationship between serum vitamin D levels with DFU.

\section{Materials and methods \\ Study selection}

Pubmed, EMBASE, BIOSIS, the Cochrane Library, and Web of Knowledge were searched for studies published before July 2018. The following combinations of search terms were used: (vitamin D, or 25-hydroxyvitamin D) AND (diabetic foot, DFU, or diabetic foot infection). There were no limitations on language and year of publication. After titles and abstracts review, studies deemed potentially eligible for inclusion were further evaluated by full text reading. Reference lists of the retrieved articles 
were also reviewed. Two reviewers (J.D. and C.J.) independently conducted the literature search, and disagreements were resolved through discussion with the third authors.

\section{Inclusion and exclusion criteria}

All eligible studies must meet the following inclusion criteria: (a) nested case-control study or cohorts study; (b) analyzing the relationship between serum vitamin $D$ levels and DFU; (c) the outcomes were serum vitamin D level or rate of severe vitamin $\mathrm{D}$ deficiency; (d) adequate data for extracting or calculating. In addition, the exclusion criteria were as follows: (a) case reports, reviews, editorials and (b) animal studies.

\section{Data extraction}

We extracted all data with a standardized datacollection protocol. For each study, two investigators independently extracted information on: information on study design, last name of the first author, publication year, country, number of patients, gender, age, serum vitamin D level, rate of severe vitamin D deficiency, and duration of follow-up.

As primary analysis, we assessed mean vitamin $\mathrm{D}$ levels in patients with DFU and in control group. As secondary analysis, we evaluated the prevalence of severe vitamin D deficiency in patients with DFU. It is widely accepted that normal 25-hydroxyvitamin D3 supplies a minimal serum value of $20 \mathrm{ng} / \mathrm{mL}$ $(50 \mathrm{nmol} / \mathrm{L})^{8}$. Serum level less than $20 \mathrm{ng} / \mathrm{mL}$ $(50 \mathrm{nmol} / \mathrm{L})$ was classified as "vitamin D deficiency" and that less than $10 \mathrm{ng} / \mathrm{mL}(25 \mathrm{nmol} / \mathrm{L})$ was defined as "severe vitamin D deficiency".

\section{Statistical analysis}

Study analyses were performed by the Cochrane Collaboration's RevMan 5.0 software. Statistical analysis of continuous variables was carried out by using mean difference (MD) with 95\% confidence intervals (CIs), and odds ratio (OR) with $95 \%$ CIs was used for dichotomous data. Serum vitamin $\mathrm{D}$ level is expressed in $\mathrm{ng} / \mathrm{mL}$ or $\mathrm{nmol} / \mathrm{L}(1 \mathrm{ng} / \mathrm{mL}=2.496 \mathrm{nmol} / \mathrm{L})$. Between-study heterogeneity was tested by $\mathrm{Q}$ statistics and $P<0.05$ indicated that there was heterogeneity present between studies. $I^{2}$ statistic method was also used and $I^{2}>50 \%$ indicated high heterogeneity.

A sensitivity analysis was performed by repeating the analysis after sequential exclusion of one study at a time.

The Newcastle-Ottawa Scale (NOS) was used to assess the quality of the included studies ${ }^{9}$. The scoring system includes three major areas: participants' selection, study comparability, and outcomes. Study with 6 or more score indicates higher quality.
Potential publication bias was evaluated by funnel plots, the Begg's rank correlation test ${ }^{10}$, and the Egger's regression test ${ }^{11}$. Data were analyzed using STATA 10.0 software. A $P$ value less than 0.05 was considered statistically significant.

\section{Results \\ Study selection and features}

A total of 143 primary studies were identified from online databases prior to July 2018. According to the inclusion and exclusion criteria, seven articles ${ }^{12-18}$ were included (Fig. 1). Six studies ${ }^{12,13,15-18}$ were published in English, and one ${ }^{14}$ was published in Russian. Both men and women were included in each study. The number of cases diagnosed in the primary studies ranged from 40 to 289 . Both men and women were included in each study. All identified studies followed an observational design, with five retrospective cohorts and two prospective cohorts. The characteristics and quality evaluation of included studies are shown in Table 1.

\section{Vitamin D levels in patients with DFU and DM groups}

Seven studies reported significantly lower serum vitamin D levels in 543 patients with DFU than in the $572 \mathrm{DM}$ group (MD $-13.47 \mathrm{nmol} / \mathrm{L}, 95 \% \mathrm{CI}-16.84$ to $-10.10, P=0.34$ ) (Fig. 2). There was no between-study heterogeneity among these studies $\left(I^{2}=12 \%, P=0.34\right)$, and sensitivity analyses showed that the summary results were not significantly influenced by any single study (Fig. 3).

\section{DFU associated with severe vitamin D deficiency}

Four studies reported data on severe vitamin D deficiency. Severe vitamin D deficiency was reported in $216(48.98 \%)$ patients with DFU and $108(22.78 \%)$ in the DM group. No substantial heterogeneity was observed $\left(I^{2}=0 \%\right)$. Based on the forest plot, severe vitamin D deficiency $(25-\mathrm{OHD}<10 \mathrm{ng} / \mathrm{mL})$ was significantly associated with the risk of DFU (OR 3.22, 95\%CI 2.42-4.28; $P=0.64, I^{2}=0 \%$ ) (Fig. 4).

The funnel plot in the meta-analysis showed no evidence of publication bias (Fig. 5). It was also indicated by Egger's test $(P=0.217)$ and Begg's test $(P=0.764)$.

\section{Discussion}

In this study, we conducted a meta-analysis of seven articles with 1115 patients, which provided a comprehensive evaluation of the association between vitamin $\mathrm{D}$ and diabetic foot. Meta-analysis reported a significantly lower vitamin D levels in patients with DFU compared with the DM group. Severe vitamin D deficiency was definitely associated with an increased risk of DFU. 

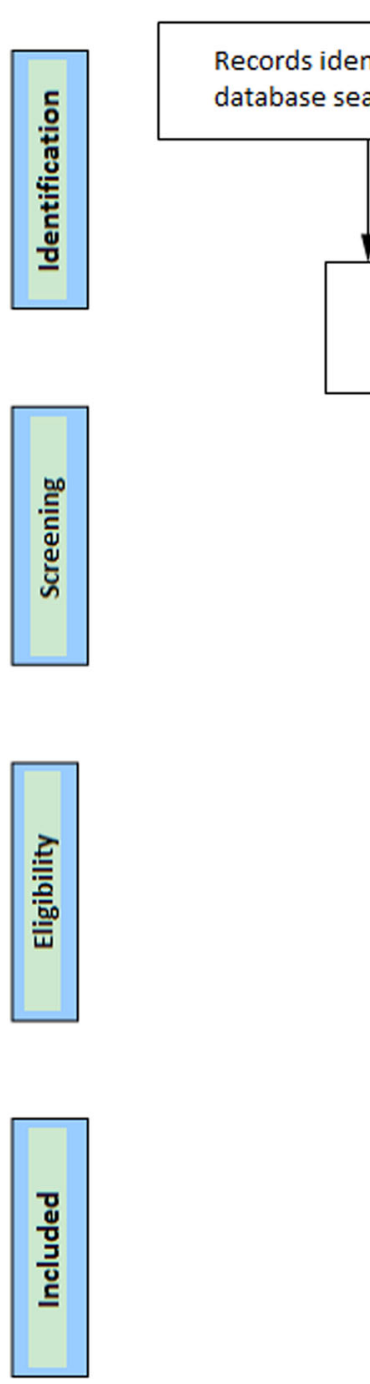

Records after duplicates removed $(n=118)$

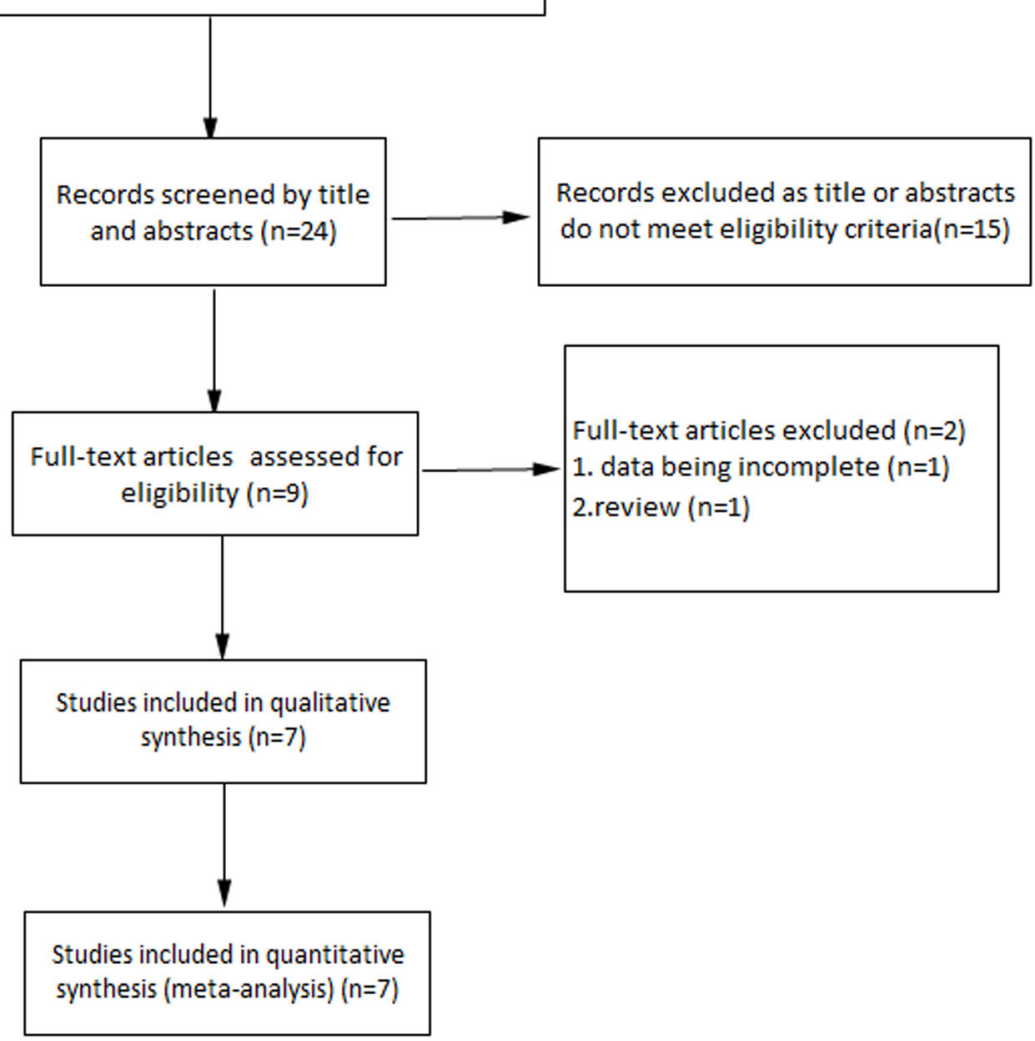

Fig. 1 Flow chart of study selection in the systematic review

Table 1 Characteristics of included studies

\begin{tabular}{|c|c|c|c|c|c|c|c|c|c|c|c|c|}
\hline & \multirow[t]{2}{*}{ Country } & \multirow[t]{2}{*}{ Study design } & \multicolumn{2}{|c|}{ Age (mean) } & \multicolumn{2}{|c|}{ Patients (No.) } & \multicolumn{2}{|c|}{ Vitamin D (nmol/L) } & \multicolumn{2}{|c|}{ Vitamin $\mathrm{D}<10 \mathrm{ng} / \mathrm{mL}$} & \multirow[t]{2}{*}{ NOS } & \multirow{2}{*}{$\begin{array}{l}\text { Vitamin D } \\
\text { assay }\end{array}$} \\
\hline & & & DFU & DM & DFU & DM & DFU & $\mathrm{DM}$ & DFU & DM & & \\
\hline Afarideh et al. ${ }^{16}$ & Iran & Prospective & 59 & 54.5 & 30 & 30 & $41.93 \pm 45.48$ & $39.94 \pm 26.07$ & NR & $N R$ & 7 & Elisa \\
\hline Feldkamp et al. ${ }^{17}$ & Germany & Retrospective & 70.2 & 69.4 & 104 & 103 & $29.45 \pm 28.2$ & $47.42 \pm 35.94$ & 57 & 32 & 8 & RIA \\
\hline Gupta and Singh ${ }^{13}$ & India & Retrospective & 54.56 & 49.62 & 50 & 50 & $35.57 \pm 21.12$ & $53.11 \pm 27.41$ & NR & $N R$ & 6 & RIA \\
\hline Ignatovich et al. ${ }^{14}$ & Russia & Prospective & NR & $N R$ & 22 & 18 & $27.75 \pm 10.25$ & $43.75 \pm 9.25$ & $N R$ & $N R$ & 7 & Elisa \\
\hline Kota et al. ${ }^{12}$ & India & Retrospective & 54.3 & 52.5 & 100 & 100 & $40.18 \pm 39.94$ & $49.42 \pm 35.19$ & 48 & 26 & 6 & RIA \\
\hline Tiwari et al..$^{15}$ & India & Retrospective & 53.6 & 51 & 125 & 164 & $40.25 \pm 38.35$ & $50.75 \pm 33$ & 57 & 28 & 6 & RIA \\
\hline Tiwari et al. ${ }^{18}$ & India & Retrospective & 53.6 & 51.9 & 112 & 107 & $40.2 \pm 39.2$ & $49.4 \pm 33.1$ & 54 & 22 & 6 & RIA \\
\hline
\end{tabular}

RIA radioimmunoassay, NR not reported, DFU diabetic foot ulcer, DM diabetes mellitus, NOS the Newcastle-Ottawa Scale 


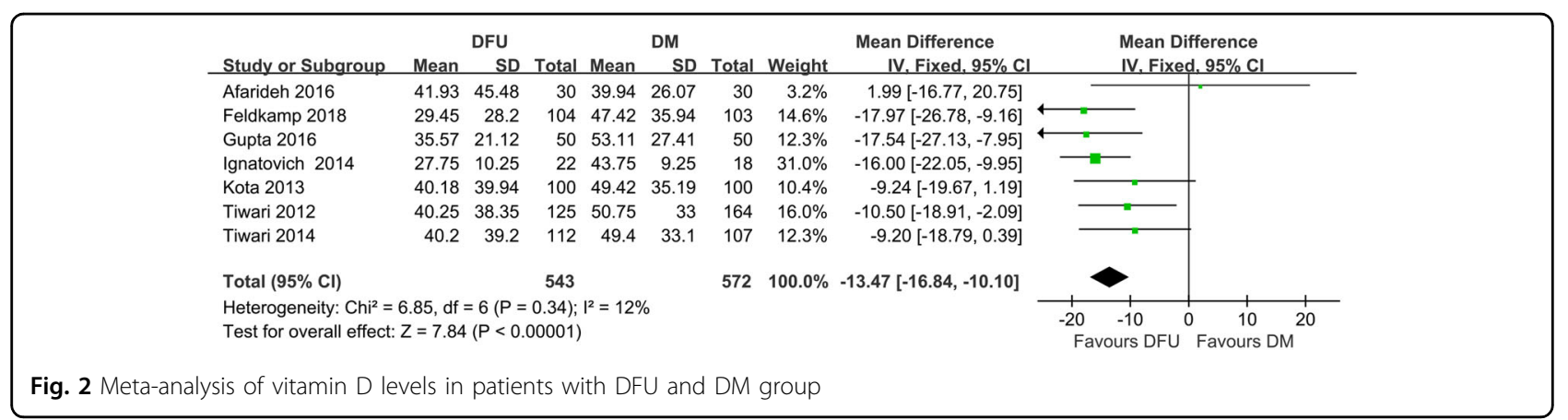

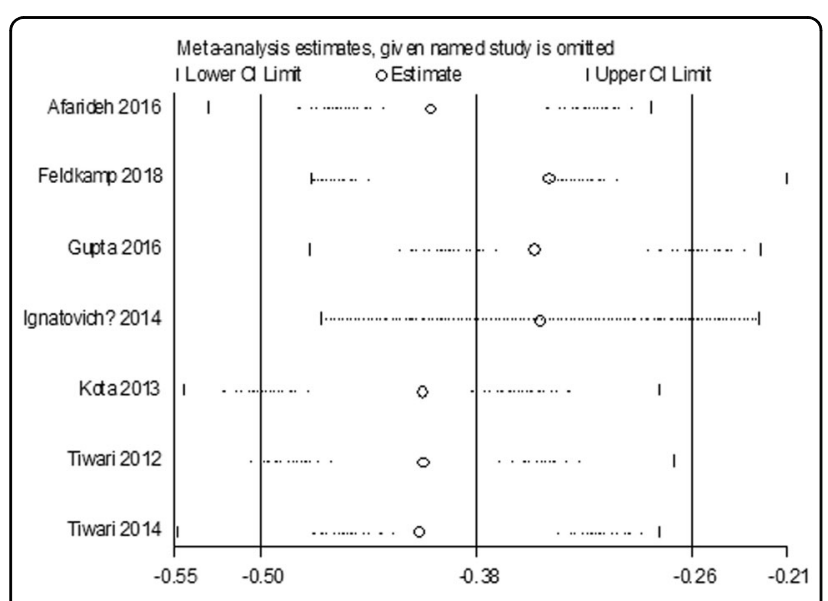

Fig. 3 Sensitivity analysis of the meta-analysis

Vitamin D has been suggested to play an important role in many chronic diseases, such as diabetes ${ }^{19}$. Low serum vitamin $\mathrm{D}$ levels are associated with insulin resistance, impaired $\beta$-cell function, and the development of $\mathrm{DM}^{20,21}$. There is also an ongoing interest in the association between lower level of vitamin $\mathrm{D}$ and diabetic complications. Zubair et al. ${ }^{22}$ found that lower $25(\mathrm{OH}) \mathrm{D}$ played a significant role in the pathogenesis of foot ulcer. Multiple mechanisms were involved in this process. Hyperglycemia in diabetic patients disturbed the normal process of cytokine production, which in turn impaired wound healing ${ }^{23}$. Vitamin D supplementation was reported to improve gylcemic control. Sugden et al. ${ }^{24}$ reported the effect of vitamin D in the improvement of endothelial function. A single high dose of oral vitamin D could substantially improve the brachial artery flow-mediated vasodilatation by $2.3 \%$. Moreover, vitamin $\mathrm{D}$ has been suggested as an immune stimulant ${ }^{25}$. Urashima et al. ${ }^{26}$ suggested that vitamin D supplementation can reduce the risk of influenza $\mathrm{A}$ in schoolchildren. Van et al. ${ }^{27}$ found vitamin D stimulated the phagocytosis and killed the intestine bacteria by macrophages. Vitamin $\mathrm{D}$ also inhibited the secretion of the $\mathrm{T}$ helper type 1 cytokines
IFN- $\gamma$ and IL-2 while stimulated the production of Th2 cytokines, which may promote wound healing ${ }^{28}$. However, the mechanism of the relationship between serum vitamin D levels and DFU is still unclear.

This study was the first meta-analysis aiming to estimate the relationship between vitamin D levels and DFU. It showed a significant reduction in vitamin $D$ levels related to DFU with a mean difference of $-13.47 \mathrm{nmol} / \mathrm{L}$ (95\%CI -16.84 to -10.10$)$. Our findings consistently supported the association between vitamin $\mathrm{D}$ levels and DFU, and also performed separate analyses for severe vitamin $\mathrm{D}$ deficiency. In the independent analyses, we found an increased incidence of DFU for declining levels of vitamin D. Patients with severe vitamin D deficiency had a higher prevalence ( 48.98 vs. $22.78 \%$ ) in diabetic foot patients, as compared with those in the DM group. Therefore, the identification of the association of DFU with vitamin D can give us some implications to develop new therapy for DFU. Vitamin D supplementation may be a valid therapeutic option for diabetes with foot ulcer and vitamin $\mathrm{D}$ deficiency. It is also supported by the only RCT that evaluated effects of vitamin D supplementation for DFU patients ${ }^{29}$. More future studies are required to verify the effect of vitamin D supplementation in the prevention or treatment of DFU.

Potential limitations of this study should be discussed. Firstly, we included only seven studies and the sample size of individual studies was small. It showed that the quantity of researched objects was insufficient. Secondly, two prospective and five retrospective cohort studies were included. Existence of bias was unavoidable in this research. In future, more well-designed trials with large number of participants are urgently required.

\section{Conclusion}

In conclusion, this is the first meta-analysis demonstrating the association between serum vitamin D levels and DFU. Severe vitamin D deficiency is significantly associated with an increased risk of DFU. More specifically designed studies are needed in the future. 


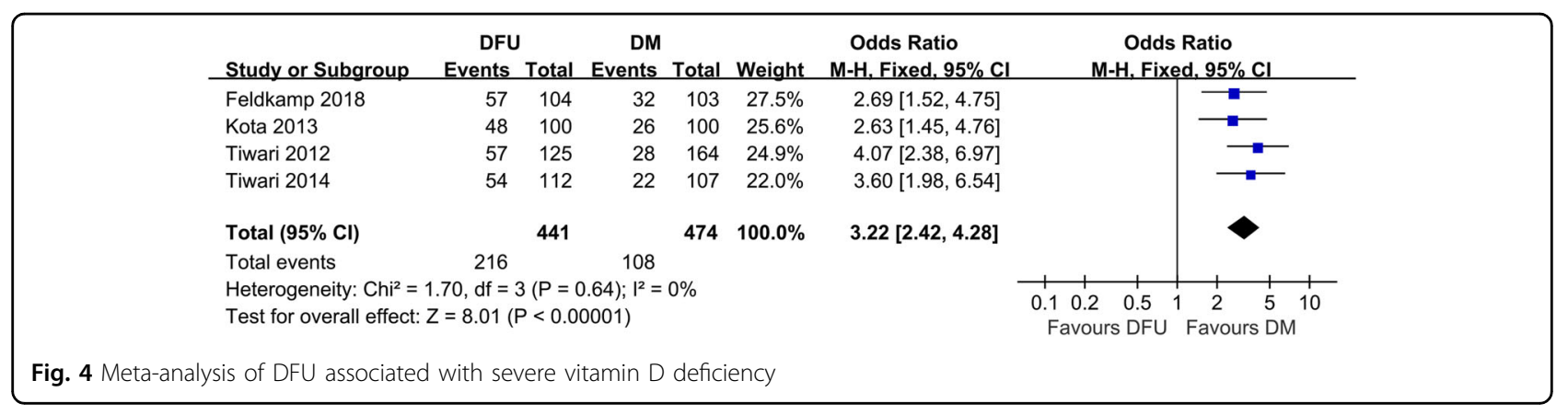

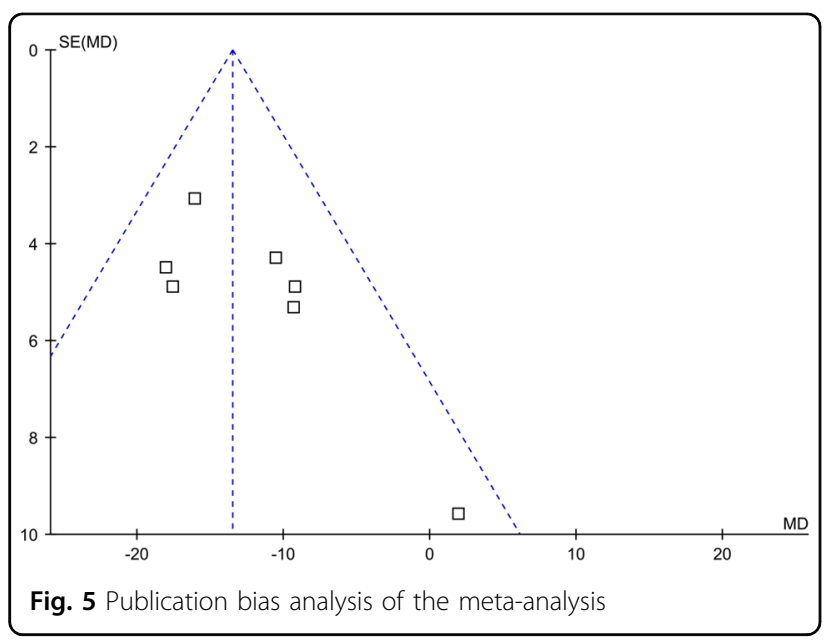

\section{Acknowledgements}

Sponsorship for this study and article processing charges were funded by the National Natural Science Foundation of China (No. 81702140) and the Interdisciplinary Program of Shanghai Jiao Tong University (YG2017QN18)

\section{Conflict of interest}

The authors declare that they have no conflict of interest.

\section{Publisher's note}

Springer Nature remains neutral with regard to jurisdictional claims in published maps and institutional affiliations.

Received: 20 August 2018 Revised: 13 February 2019 Accepted: 25 February 2019

Published online: 11 March 2019

\section{References}

1. Armstrong, D. G. et al. Effect of oral nutritional supplementation on wound healing in diabetic foot ulcers: a prospective randomized controlled trial. Diabet. Med. 31, 1069-1077 (2014).

2. Daousi, C. et al. Chronic painful peripheral neuropathy in an urban community: a controlled comparison of people with and without diabetes. Diabet. Med. 21, 976-982 (2004).

3. Asemi, Z., Hashemi, T., Karamali, M., Samimi, M. \& Esmaillzadeh, A. Effects of vitamin D supplementation on glucose metabolism, lipid concentrations, inflammation, and oxidative stress in gestational diabetes: a double-blind randomized controlled clinical trial. Am. J. Clin. Nutr. 98, 1425-1432 (2013).
4. Yakob, M. A. H., Leong, J. F. \& Pande, K. C. Vitamin D and other biochemical markers of nutrition in patients with diabetic foot infection in negara brunei darussalam. In IOF Regionals—5th Asia-Pacific Osteoporosis Meeting Osteoporos Int 25, 639 (2014).

5. Gonzalez-Curiel, I. et al. 1,25-dihydroxyvitamin D3 induces LL-37 and HBD-2 production in keratinocytes from diabetic foot ulcers promoting wound healing: an in vitro model. PLOS ONE 9, e111355 (2014).

6. Tian, X. Q.. Chen, T. C. \& Holick, M. F. 1,25-dihydroxyvitamin D3: a novel agent for enhancing wound healing. J. Cell. Biochem. 59, 53-56 (1995).

7. Trujillo, V. et al. Calcitriol promotes proangiogenic molecules in keratinocytes in a diabetic foot ulcer model. J. Steroid Biochem. Mol. Biol. 174, 303-311 (2017).

8. Hollis, B. W. Assessment of vitamin D status and definition of a normal circulating range of 25-hydroxyvitamin D. Curr. Opin. Endocrinol. Diabetes Obes. 15, 489-494 (2008).

9. Wells, G. A. et al. The Newcastle-Ottawa Scale (NOS) for assessing the quality of non-randomized studies in meta-analysis. Appl. Eng. Agric. 18, 727-734 (2014).

10. Begg, C. B. \& Mazumdar, M. Operating characteristics of a rank correlation test for publication bias. Biometrics 50, 1088-1101 (1994).

11. Egger, M., Davey Smith, G., Schneider, M. \& Minder, C. Bias in meta-analysis detected by a simple, graphical test. BMJ 315, 629-634 (1997).

12. Kota, S. K. Meher, L. K., Jammula, S. \& Modi, K. D. Inflammatory markers in diabetic foot and impact of vitamin D deficiency. Endocrine Abst. 31, 198 (2013).

13. Gupta, B. \& Singh, S. K. Invitro study of role of vitamin d on macrophages dysfunction in patients with diabetic foot infection. Int. J. Adv. Res. 4, 1633-1637 (2016).

14. Ignatovich, I. N., Kondratenko, G. G. \& Luv, D. S. [Low level of 25-OH-vitamin D as a marker of critical ischemia in case of diabetic foot syndrome]. Khirurgiia. $11-14$ (2014).

15. Tiwari, S. et al. Prevalence and severity of vitamin $D$ deficiency in patients with diabetic foot infection. Br. J. Nutr. 109, 99-102 (2013).

16. Afarideh, M. et al. Raised serum 25-hydroxyvitamin D levels in patients with active diabetic foot ulcers. Br. J. Nutr. 115, 1938-1946 (2016).

17. Feldkamp, J., Jungheim, K., Schott, M., Jacobs, B. \& Roden, M. Severe vitamin D3 deficiency in the majority of patients with diabetic foot ulcers. Horm. Metab. Res. 50, 615-619 (2018).

18. Tiwari, S., Pratyush, D. D., Gupta, S. K. \& Singh, S. K. Vitamin D deficiency is associated with inflammatory cytokine concentrations in patients with diabetic foot infection. Br. J. Nutr. 112, 1938-1943 (2014).

19. Lee, C. J. et al. The effect of vitamin D supplementation on glucose metabolism in type 2 diabetes mellitus: a systematic review and meta-analysis of intervention studies. J. Diabetes Complicat. 31, 1115-1126 (2017).

20. Hahn, S. et al. Low serum 25-hydroxyvitamin D concentrations are associated with insulin resistance and obesity in women with polycystic ovary syndrome. Exp. Clin. Endocrinol. Diabetes 114, 577-583 (2006).

21. Akbari, M. et al. The effects of vitamin D supplementation on glucose metabolism and lipid profiles in patients with gestational diabetes: a systematic review and meta-analysis of randomized controlled trials. Horm. Metab. Res. 49, 647-653 (2017).

22. Zubair, M., Malik, A., Meerza, D. \& Ahmad, J. 25-Hydroxyvitamin D [25(OH)D levels and diabetic foot ulcer: is there any relationship? Diabetes Metab. Syndr. Clin. Res. Rev. 7, 148-153 (2013). 
23. Naguib, G., Al-Mashat, H., Desta, T. \& Graves, D. T. Diabetes prolongs the inflammatory response to a bacterial stimulus through cytokine dysregulation. J. Invest. Dermatol. 123, 87-92 (2004).

24. Sugden, J. A., Davies, J. I., Witham, M. D., Morris, A. D. \& Struthers, A. D. Vitamin $D$ improves endothelial function in patients with Type 2 diabetes mellitus and low vitamin D levels. Diabet. Med. 25, 320-325 (2008).

25. Greenhagen, R. Serum vitamin D and the diabetic foot. Podiatry Manag. 30, 163 (2011).

26. Urashima, M. et al. Randomized trial of vitamin D supplementation to prevent seasonal influenza A in schoolchildren. Am. J. Clin. Nutr. 91, 1255-1260 (2010).
27. Van, E. E., Decallonne, B., Bouillon, R. \& Mathieu, C. NOD bone marrow-derived dendritic cells are modulated by analogs of 1,25-dihydroxyvitamin D3. J. Steroid Biochem. Mol. Biol. 89-90, 457 (2004).

28. van Etten, E. \& Mathieu, C. Immunoregulation by 1,25-dihydroxyvitamin D3: basic concepts. J. Steroid Biochem. Mol. Biol. 97, 93-101 (2005).

29. Razzaghi, R. et al. The effects of vitamin D supplementation on wound healing and metabolic status in patients with diabetic fot ulcer: a randomized, double-blind, placebo-controlled trial. J. Diabetes Complicat. 31, 766-772 (2017). 